\title{
Investigation of a Possible Association Between Dynamic Thiol/Disulfide Homeostasis and Pain in Knee Arthroplasty Patients
}

\author{
Hicabi Sezgin ${ }^{1}$, Bilal İlanbey ${ }^{2}$ \\ ${ }^{1}$ Department of Orthopedics and Traumatology, Amasya University School of Medicine, Amasya, Turkey \\ ${ }^{2}$ Department of Biochemistry, Ahi Evran University School of Medicine, Kirsehir, Turkey
}

Received: 23 October 2020, Accepted: 03 December 2020, Published online: 31 December 2020

(C) Ordu University Institute of Health Sciences, Turkey, 2020

\begin{abstract}
Objective: Dynamic thiol/disulfide homeostasis plays a pivotal role in many physiologic mechanisms. In the present study, we aimed to elucidate the relationship between postoperative pain and thiol/disulfide homeostasis in patients who underwent primary total knee arthroplasty for primary knee osteoarthritis.

Methods: This prospective uncontrolled study included 28 patients (10 male, 18 female) with a diagnosis of primary gonarthrosis who underwent primary total knee arthroplasty and met the study inclusion criteria. Venous blood samples were taken from the patients and pain values were recorded simultaneously using the visual analog scale (VAS) preoperatively and at the 4th postoperative week. Thiol/disulfide hemostasis levels were measured using a new fully-automated and spectrophotometric method developed by Erel and Neselioglu.

Results: The average age of the patients participating in the study was $66.25 \pm 8.29$ years and $18(64.3 \%)$ of the patients were female. B values for preoperative native/total thiol $(+7,652)$, preoperative disulfide/native thiol $(+10,550)$, and postoperative total thiol $(+128)$, positively affected the difference in VAS values. B values for preoperative disulfide $(-219)$, postoperative disulfide $(-1,297)$, postoperative native/total thiol ($4,238)$, and postoperative disulfide/native thiol $(-3,316)$ negatively affected the difference in VAS values.

Conclusion: The disulfide level, an oxidized form in thiol/disulfide homeostasis, appears to be a valuable marker for evaluating the effect of oxidative stress on postoperative pain.
\end{abstract}

Key words: Thiol/disulfide homeostasis, oxidative stress, pain, osteoarthritis, knee arthroplasty

Suggested Citation: Sezgin H, Ilanbey B. Investigation of a Possible Association Between Dynamic Thiol/Disulfide Homeostasis and Pain in Knee Arthroplasty Patients. Middle Black Sea Journal of Health Science, 2020; 6(3):352-357.

\section{Address for correspondence/reprints:}

Hicabi Sezgin

Telephone number: +90 (358) 2184000

ORCID-ID 0000-0002-1332-3656

E-mail: hicabi.sezgin@ @otmail.com

DOI: $10.19127 / \mathrm{mbsjohs.815483}$

\section{Introduction}

Osteoarthritis (OA), the most well-known form of arthritis, is defined by degeneration of the articular cartilage. As life expectancy has increased, OA has become a common disease in the elderly. Oxidative stress, described as an imbalance between increased free radical load and cellular clearance mechanisms is an important part of OA pathogenesis (Ziskoven et al., 2010).

Total knee arthroplasty (TKA) is a surgical procedure applied to patients with severe knee OA to obtain a painless functional joint and increase their 
quality of life. Pain that occurs after TKA is a major concern for patients and may directly affect functional results. It has been shown that oxidative stress may lead to hyperalgesia by causing peripheral sensitization and inflammation (Nashed, Balenko, \& Singh, 2014; Ergonenc \& Beyaz, 2019).

Thiols, organic molecules including sulfhydryl groups, are the basis of the oxidant/antioxidant balance in the body. When thiols (the reduced state) undergoes an oxidative reaction, they turn into disulfide groups (the oxidized state). Disulfide groups can be degraded back to thiol groups increasing thiol reserves. Dynamic thiol/disulfide homeostasis is provided because of these reactions at the cellular level. Dynamic thiol/disulfide homeostasis plays an essential role in antioxidant guard, detoxification, cell signaling and transcription, apoptosis, and regulation of enzymatic activity. Studies have revealed that in thiol/disulfide homeostasis, an increase in the balance towards thiols leads to proliferation, while an increase towards disulfides leads to apoptosis (Nar \& Calis, 2018). Disulfide levels were found to be higher in patients with degenerative states such as smoking, diabetes, obesity, and OA, and lower in patients with proliferative conditions such as multiple myeloma and cancers. Erel and Neselioglu described a new method to determine dynamic thiol/disulfide homeostasis which allowed them to evaluate native and total thiol concentrations simultaneously. The oxidant-antioxidant balance can be assessed together with disulfide parameters calculated handling native thiol and total thiol levels (Erel \& Neselioglu, 2014).

In the current study, we proposed to elucidate the correlation between postoperative pain and thiol/disulfide homeostasis in patients who underwent primary TKA for primary knee OA. There is no previous study investigating the relationship between pain and thiol/disulfide homeostasis after TKA.

\section{Methods}

\section{Study design}

This prospective cohort study was carried out with the approval of the Ahi Evran University Clinical Research Ethics Committee (2019-05/61). This study was supported by the Kursehir Ahi Evran University Scientific Research Project Unit (TIP.A4.19.002) and prepared in accordance with the Helsinki Declaration of Principles. This study has not been presented as a poster or oral presentation previously.

The present study included 28 patients (10 males, 18 females) with a diagnosis of primary gonarthrosis who underwent primary TKA between April 2019 and March 2020 at the Kursehir Ahi Evran University Medical Faculty Orthopedics and Traumatology Clinic and met the inclusion criteria. Patients with comorbidities such as diabetes mellitus, cardiovascular disease, acute or chronic renal failure, hyperlipidemia, depression, chronic lung disease, inflammatory disease, malignancy, or infection were not included in the study. In addition, individuals using any medication (steroidal or non-steroidal antiinflammatory drugs, antioxidants, vitamin supplements) during the 4 weeks before venous blood sampling were also excluded. Study patients also did not smoke or consume alcohol.

All surgical procedures were performed under spinal anesthesia by the same surgeon experienced in the field, using the same surgical technique and medial parapatellar approach. In all cases, a lower extremity pneumatic tourniquet was adjusted to a pressure of $300 \mathrm{mmHg}$ during the operation. The same brand and type of cemented posterior-stabilized primary knee prosthesis (Vanguard Rotating Platform High Flex; Biomet, Warsaw, IN, USA) was applied for all 28 patients. Patellofemoral surface change was not applied, and desensitization was performed around the patella with electrocautery. A drainage tube was not used in the operation. The same drug protocol routinely used after knee arthroplasty in our clinic was applied for 5 days (paracetamol $3 \times 1 \mathrm{gr}$ iv, cefazolin $3 \times 1 \mathrm{gr}$ iv, tramadol $3 \times 100 \mathrm{mg}$ iv, pantoprazole $1 \times 40 \mathrm{mg}$ iv, enoxaparin $1 \times 0.4 \mathrm{ml} \mathrm{sc}$ ). Steroidal or non-steroidal anti-inflammatory drug treatment was not used. The patients were mobilized by placing full weight at the 24 th postoperative hour and the same rehabilitation program was applied for all patients in the presence of a physiotherapist.

At 24 hours preoperatively and during the 4th postoperative week, after fasting overnight, $3 \mathrm{ml}$ of venous blood was taken from the patients and transferred to ethylenediaminetetraacetic acid (EDTA) containing tubes. The collected samples were centrifuged at $4^{\circ} \mathrm{C}$ and $1500 \times \mathrm{g}$ for 10 minutes. The plasma samples were kept frozen at $-80^{\circ} \mathrm{C}$ until the thiol/disulfide homeostasis analysis tests were conducted at which time all samples were thawed and analyzed simultaneously.

The pain values of the patients were recorded simultaneously with the preoperative and postoperative venous blood samples, using the visual analog scale (VAS) scale. VAS is a widely used scale for evaluating pain severity. The scale ranges from 0 to 10 , with 0 indicating no pain and a value of 10 meaning unbearable pain (Karcioglu, Topacoglu, Dikme, \& Dikme, 2018). 


\section{Biochemical Analysis}

Thiol/disulfide hemostasis levels were calculated using the spectrophotometric method developed by Erel and Neselioglu(Erel \& Neselioglu, 2014). A Shimadzu UV-1800 spectrophotometer with a temperature-controlled cuvette holder and Cobas c501 automatic analyzer (Roche) were used.

After defining the native and total thiol levels, the disulfide concentration was determined using the following formula:

Disulfide $=($ total thiol-native thiol $) / 2$

Disulfide/total thiol (\%), disulfide/native thiol $(\%)$, and native thiol/total thiol (\%) ratios were calculated using previously determined disulfide, native thiol, and total thiol concentrations (Erel, 2005).

\section{Statistical Analyses}

The skewness and kurtosis values between -3 and +3 , sufficient for normal distribution and parametric methods, were used to evaluate the results of the study. The relationship of variables was evaluated using t-tests and parametric tests. The interaction between dependent (VAS score) and independent variables (native thiol, total thiol, disulfide, disulfide/native thiol, disulfide/total thiol, native thiol/total thiol) was investigated using the multiple linear regression method. $p<0.05$ was noted statistically significant. SPSS version 22 for Windows (SPSS Inc., Chicago, IL, USA) was used to evaluate the results.

\section{Results}

The average age of the patients participating in the study was $66.25 \pm 8.29$ years and $18(64.3 \%)$ of the patients were female. All of the 28 cases involved TKA performed due to primary OA. No local or systemic complications were observed in the patients during the postoperative period. No peri-operative blood transfusions were required. While the preoperative mean C-reactive protein (CRP) value was $0.4 \pm 0.4$, the postoperative 4 th week CRP mean value increased to $1.4 \pm 1.1$, which was a statistically significant difference between the preoperative and postoperative CRP measurements ( $\mathrm{p}<0.05$ ).

Pre- and postoperative patient VAS, native thiol, total thiol, disulfide, disulfide/native thiol, disulfide/total thiol, and native thiol/total thiol values are given in Table1. The difference between the preand postoperative VAS measurements is statistically significant $(p<0.05)$ (Table 2). Linear regression analysis was applied to examine the parameters affecting this difference between the pre- and postoperative VAS measurements (Table 3). According to this analysis method, among the independent variables it was found that the rates of preoperative native/total thiol, preoperative disulfide/native thiol, and postoperative total thiol, positively affected the VAS difference (B values: $+7,652,+10,550$, and +128 , respectively) whereas preoperative disulfide, postoperative disulfide, postoperative native/total thiol, and postoperative disulfide/native thiol ratios negatively affected the difference in VAS scores (B values: -219, -1,297, 4,238 , and $-3,316$, respectively) (Table 3 ). In other words, lower preoperative disulfide, postoperative disulfide values, and postoperative native/total thiol, and postoperative disulfide/native thiol ratios correlated with lower patient postoperative VAS scores

$\underline{\text { Table 1. Pre- and postoperative patient variables }}$

\begin{tabular}{lll}
\hline & $\begin{array}{l}\text { Preoperative } \\
\text { Mean } \pm \text { SD }\end{array}$ & $\begin{array}{l}\text { Postoperative } \\
\text { Mean } \pm \text { SD }\end{array}$ \\
\hline VAS & $7.6 \pm 2.1$ & $3.2 \pm 0.9$ \\
Native Thiol $(\mu \mathrm{mol} / \mathrm{L})$ & $218.0 \pm 53.0$ & $223.6 \pm 38.7$ \\
Total Thiol $(\mu \mathrm{mol} / \mathrm{L})$ & $261.8 \pm 59.5$ & $264.6 \pm 40.7$ \\
Disulfide $(\mu \mathrm{mol} / \mathrm{L})$ & $21.9 \pm 4.6$ & $20.5 \pm 5.4$ \\
Disulfide/Native Thiol $(\%)$ & $10.3 \pm 1.8$ & $9.4 \pm 2.7$ \\
Disulfide/Total Thiol $(\%)$ & $8.5 \pm 1.3$ & $7.8 \pm 1.9$ \\
Native Thiol/Total Thiol $(\%)$ & $83.0 \pm 2.5$ & $84.3 \pm 3.9$ \\
\hline
\end{tabular}

Table 2. Pre and postoperative VAS scores

\begin{tabular}{|c|c|c|c|}
\hline & $\begin{array}{c}\text { Preoperative } \\
\text { Mean } \pm \text { SD }\end{array}$ & $\begin{array}{c}\text { Postoperative } \\
\text { Mean } \pm \text { SD }\end{array}$ & $\mathrm{p}$ \\
\hline VAS & $7.6 \pm 2.1$ & $3.2 \pm 0.9$ & $.000 *$ \\
\hline
\end{tabular}


Table 3. Linear regression analysis of variables

\begin{tabular}{lllll}
\hline Dependent Variable & Independent Variable & B & p & $\mathrm{R}^{2}$ \\
\hline & Pre-op Disulfide & -.219 & $.016^{*}$ \\
& Pre-op Native/Total Thiol $(\%)$ & 7,652 & $.006^{*}$ \\
& Pre-op Disulfide/Native Thiol (\%) & 10,550 & $.006^{*}$ \\
Pre-op-Post-op VAS Gap & Post-op Total Thiol & .128 & $.029 *$ & .668 \\
& Post-op Disulfide & $-1,297$ & $.050 *$ & $.042^{*}$ \\
& Post-op Native/Total Thiol $(\%)$ & $-4,238$ & .094 \\
\hline
\end{tabular}

$* \mathrm{p}<0.05$ is considered statistically significant.

\section{Discussion}

In the present study, it was observed that patients with low levels of disulfide, which is an indicator of oxidative stress levels, had lower postoperative VAS scores; that is, they felt less pain. There are several publications in the literature describing the pathophysiological connection between oxidative stress levels and pain. One study indicated that oxidative stress could trigger pain pathways through glutamatergic signaling and activation of inflammatory reactions, as well as by directly affecting nociceptive centers in the brain (Perrone et al., 2017). In another study, it was mentioned that oxidative stress may cause peripheral sensitization; that is, it can change nociception that may cause hyperalgesia (Nashed et al., 2014). Therefore, it has been recommended that a reduction of oxidative stress by antioxidants may reduce peripheral inflammation and stimulation of nociceptive receptors and alleviate pain. In support of this mechanism, Payal et al. (2009) showed that antioxidant supplementation reduced oxidative stress levels and alleviated pain levels in patients with chronic pancreatitis. Bellieni et al. (2009) observed a positive correlation between high pain levels and high oxidative stress levels in newborns. In another study conducted with ankylosing spondylitis patients, it was reported that native and total thiol levels negatively correlated with Bath Ankylosing Spondylitis Disease Activity Index (BASDAI) and VAS scores (Dogru et al., 2016). Ayhan Tuzcu et al.(Tuzcu et al., 2019) examined thiol/disulfide homeostasis in patients with fibromyalgia syndrome (FMS) and found that native thiol and native thiol/total thiol levels were significantly lower in FMS cases compared to healthy individuals; however, disulfide levels in FMS patients were significantly higher than in healthy individuals. They also found a positive correlation between disulfide levels and pain and the number of sensitive points (Tuzcu et al., 2019).

In current study, similar to other studies in the literature, a significant relationship was found between low levels of disulfide and low VAS scores. In support of this relationship statistically, the preoperative native/total thiol ratio positively affected the pre- and postoperative VAS score difference. However, contrary to expectations, the postoperative native/total thiol ratio negatively affected the pre- and postoperative VAS score difference. According to the literature, this is not an expected result. Similarly, many articles in the literature on oxidative stress have shown conflicting data. Inanir et al. (Inanır, Sogut, Ayan, \& Inanır, 2013), in their study to evaluate the relationship between pain level and oxidative stress levels in patients with acute and chronic inflammatory or noninflammatory low back pain, observed that there was no relationship between oxidative stress levels and pain intensity and pain level.

As in the present study, other authors have attempted to set a link between oxidative stress and disease pathophysiology. It is hard to evaluate oxidative stress and the response of the human body to this stress because there is no single biomarker that can objectively measure oxidative stress (Grune \& Berger, 2007). In addition, there is no biomarker showing the tissue oxidative stress level in peripheral blood (Arsalani-Zadeh, Ullah, Khan, \& MacFie, 2011). It has been shown that oxidative damage can appear at the tissue level without a measurable change in peripheral blood (Kerkweg et al., 2010). Also, blood and tissue biomarkers of oxidative stress may not follow the same pattern of change (Arguelles, Garcia, Maldonado, Machado, \& Ayala, 2004). In the current study, we found that the preoperative native/total thiol ratio positively affected the pre- and postoperative VAS score difference, but contrary to 
what was expected, the postoperative native/total thiol ratio negatively affected the pre- and postoperative VAS score difference, which may be explained by that situation. In light of the data obtained, we think that the disulfide level, an oxidized form, is a more valuable marker than postoperative native/total thiol ratio in evaluating the effect of oxidative stress on postoperative pain.

Due to the large number of exclusion criteria, the low number of patients meeting the inclusion criteria is one of the most prominent limitations of the current study. Randomized controlled studies with large patient populations are required to obtain statistically significant data. In addition to the absence of an ideal method for measuring oxidative stress, the unknown clinical significance of oxidative stress changes can be considered as the main limitation of oxidative stress measurement studies in general.

\section{Conclusion}

We think that the disulfide level, an oxidized form, is a more valuable marker than other independent variables evaluating the effect of oxidative stress on both postoperative and preoperative pain

Ethics Committee Approval: This study was approved by the Ethics Committee of the Ahi Evran University Clinical Research Ethics Committee (Ethical approval number: Protocol Number 201905/61) and prepared in accordance with the Helsinki Peer-review: Externally peer-reviewed.

Author Contributions: Concept -HS, BI; DesignHS,BI; Supervision-HS; Materials - HS, BI; Data Collection and/or Processing -HS.; Analysis and/or Interpretation -HS, BI; Literature Review -HS; Writing - HS; Critical Review - HS, BI.

Conflict of Interest: No conflict of interest was declared by the authors.

Financial Disclosure: This study was supported by the Kursehir Ahi Evran University Scientific Research Project Unit (TIP.A4.19.002)

\section{References}

Arguelles, S., Garcia, S., Maldonado, M., Machado, A., \& Ayala, A. (2004). Do the serum oxidative stress biomarkers provide a reasonable index of the general oxidative stress status? Biochimica et Biophysica Acta, 1674(3), 251-259. doi:10.1016/j.bbagen.2004.06.023
Arsalani-Zadeh, R., Ullah, S., Khan, S., \& MacFie, J. (2011). Oxidative stress in laparoscopic versus open abdominal surgery: a systematic review. Journal of Surgical Research, 169(1), e59-68. doi:10.1016/j.jss.2011.01.038

Bellieni, C. V., Iantorno, L., Perrone, S., Rodriguez, A., Longini, M., Capitani, S., \& Buonocore, G. (2009). Even routine painful procedures can be harmful for the newborn. Pain, 147(1-3), 128-131. doi:10.1016/j.pain.2009.08.025

Bhardwaj, P., Garg, P. K., Maulik, S. K., Saraya, A., Tandon, R. K., \& Acharya, S. K. (2009). A randomized controlled trial of antioxidant supplementation for pain relief in patients with chronic pancreatitis. Gastroenterology, 136(1), 149-159 e142. doi:10.1053/j.gastro.2008.09.028

Dogru, A., Balkarli, A., Cetin, G. Y., Neselioglu, S., Erel, O., Tunc, S. E., \& Sahin, M. (2016). Thiol/disulfide homeostasis in patients with ankylosing spondylitis. Bosnian Journal of Basic Medical Sciences. Udruzenje Basicnih Mediciniskih Znanosti, 16(3), 187-192. doi:10.17305/bjbms.2016.1001

Erel, O. (2005). A new automated colorimetric method for measuring total oxidant status. Clinical Biochemistry, 38(12), 1103-1111. doi:10.1016/j.clinbiochem.2005.08.008

Erel, O., \& Neselioglu, S. (2014). A novel and automated assay for thiol/disulphide homeostasis. Clinical Biochemistry, 47(18), 326-332. doi:10.1016/j.clinbiochem.2014.09.026

Ergonenc, T., \& Beyaz, S. G. (2019). T Long-term Effects of Ultrasound-guided Genicular Nerve Pulsed Radiofrequency on Pain and Knee Functions in Patients with Gonarthrosis. . J Sakarya Tip Dergis, 9(1), 52-58. doi:10.31832/smj.506472

Grune, T., \& Berger, M. M. (2007). Markers of oxidative stress in ICU clinical settings: present and future. Current Opinion in Clinical Nutrition and Metabolic Care, 10(6), 712-717. doi:10.1097/MCO.0b013e3282f0c97c

Inanır, A., Sogut, E., Ayan, M., \& Inanır, S. (2013). Evaluation of Pain Intensity and Oxidative Stress Levels in Patients with Inflammatory and NonInflammatory Back Pain. Eur J Gen Med., 10(4), 185-190. doi:doi.org/10.29333/ejgm/82205

Karcioglu, O., Topacoglu, H., Dikme, O., \& Dikme, O. (2018). A systematic review of the pain scales in adults: Which to use? American Journal of Emergency Medicine, 36(4), 707-714. doi:10.1016/j.ajem.2018.01.008 
Kerkweg, U., Pamp, K., Fieker, J., Petrat, F., Hider, R. C., \& de Groot, H. (2010). Release of redoxactive iron by muscle crush trauma: no liberation into the circulation. Shock, 33(5), 513-518. doi:10.1097/SHK.0b013e3181c4f56e

Nar, R., \& Calis, A. G. (2018). Assessment of dynamic thiol/disulfide homeostasis in patients with asthma. Journal of Laboratory Medicine, 42(3), 99. doi:https://doi.org/10.1515/labmed2017-0144

Nashed, M. G., Balenko, M. D., \& Singh, G. (2014). Cancer-induced oxidative stress and pain. Curr Pain Headache Rep, 18(1), 384. doi:10.1007/s11916-013-0384-1

Perrone, S., Bellieni, C. V., Negro, S., Longini, M., Santacroce, A., Tataranno, M. L., . . Buonocore, G. (2017). Oxidative Stress as a Physiological Pain Response in Full-Term Newborns. Oxidative Medicine and Cellular Longevity, 2017, 3759287. doi:10.1155/2017/3759287

Tuzcu, A., Baykara, R. A., Alisik, M., Omma, A., Acet, G. K., Dogan, E., .Erel, O. (2019). Alteration of Thiol-Disulfide Homeostasis in Fibromyalgia Syndrome. Acta Medica (Hradec Kralove), 62(1), 12-18. doi:10.14712/18059694.2019.40

Ziskoven, C., Jager, M., Zilkens, C., Bloch, W., Brixius, K., \& Krauspe, R. (2010). Oxidative stress in secondary osteoarthritis: from cartilage destruction to clinical presentation? Orthopedic Reviews, 2(2), e23. doi:10.4081/or.2010.e23 